\title{
Beam Spin Asymmetry Measurements from Deeply Virtual Meson Production
}

\author{
K. Joo*, R. De Masi ${ }^{\dagger}$, M. Garçon ${ }^{\dagger}$, V. Kubarovsky**, P. Stoler**, M. \\ Ungaro* and B. Zhao* \\ ${ }^{*}$ University of Connecticut, Storrs, CT, U.S.A. \\ ${ }^{\dagger}$ CEA-Saclay, Service de Physique Nucléaire, Gif-sur-Yvette, France \\ ${ }^{* *}$ Rensselaer Polytechnic Institute, Troy, NY, USA
}

\begin{abstract}
Study of deeply virtual exclusive meson production (DVMP), is being conducted in the E1-DVCS experiment with the CLAS detector at Jefferson Lab. The main motivation of the experiment is to characterize the partonic properties of the nucleon in the framework of generalized parton distributions (GPDs). The data were taken in the spring of 2005 using a $5.7 \mathrm{GeV}$ longitudinally polarized electron beam and an unpolarized hydrogen target. We report on the on-going beam spin asymmetry analysis for pseudo-scalar channels and future experiments.
\end{abstract}

Keywords: GPDs, hard exclusive processes

PACS: $13.60 .-r, 24.85 .+p, 25.30 . D h$

About ten years ago, a unifying concept for the description of the nucleon structure was introduced, now commonly known as generalized parton distributions (GPDs) [1, 2, $3,4,5,6]$. These functions contain the usual form factors and parton distributions, but in addition they include correlations between states of different longitudinal momentum and transverse momentum dependence. GPDs can therefore give three-dimensional pictures of the nucleon, providing information such as the transverse spatial distribution as a function of the longitudinal momentum fraction of quarks.

There are four independent (chiral-even) GPDs: $H, \tilde{H}, E$, and $\tilde{E} . H$ and $E$ are so-called spin-independent, and $\tilde{H}$ and $\tilde{E}$ spin-dependent functions. In the forward direction, $H$ reduces to the quark distribution $q(x)$, and $\tilde{H}$ to the quark-helicity distribution $\Delta q(x)$ measured in deep inelastic scattering. Furthermore, at finite momentum transfer, there are model independent sum rules that relate the first moments of these GPDs to the standard hadronic form factors. Also, Ji [2] has shown that the second moment of these GPDs gives access to the contribution of the sum of the quark spin and the quark orbital angular momentum to the nucleon spin.

The GPDs reflect the structure of the nucleon independently of the reaction that probes the nucleon. They can be accessed by deeply virtual meson productions (DVMP) as well as deeply virtual Compton scattering (DVCS). The complete extraction of the GPDs requires an extensive program rather than a single experiment, involving the measurements of a variety of channels and observables over a broad kinematic range. For example, the main contributions to the beam spin asymmetries (BSA or $A_{L U}$ ) of DVCS are due to $H$ whereas the main contributions to the target spin asymmetries (TSA or $A_{U L}$ ) of DVCS come from both $H$ and $\tilde{H}$. To access the GPD $E$, which contains direct information on the

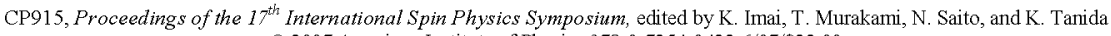


orbital angular momentum of the quarks, a transversely polarized target is needed. On the other hand, leading-order $\mathrm{pQCD}$ predicts that the vector meson channels are sensitive only to the unpolarized GPDs ( $H$ and $E$ ), whereas the pseudoscalar meson channels are mostly sensitive to the polarized GPDs $(\tilde{H}$ and $\tilde{E})$. Especially $\pi^{0}$ and $\eta$ channels are mostly sensitive to $\tilde{H}$. Ultimately a global analysis will be required to extract the GPDs from a large set of measurements.

There are two Jefferson Lab CLAS experiments, E01-113 [7] and E05-114 [8], which are dedicated to study GPDs by measuring hard exclusive processes such as DVCS and DVMP. E01-113 is to measure $A_{L U}$ using a polarized electron beam and an unpolarized hydrogen target, and E05-114 to measure $A_{U L}$ using an unpolarized electron beam and a longitudinally polarized hydrogen target. These observables are linked to specific linear combinations of generalized parton distributions (GPDs). Kinematic coverages of DVCS and DVMP are very similar. These measurements will provide a full mapping of $A_{L U}$ and $A_{U L}$ for DVCS and DVMP over the available $x_{B}, t$ and $\mathrm{Q}^{2}$ range from CEBAF's $5.7 \mathrm{GeV}$ electron beam.

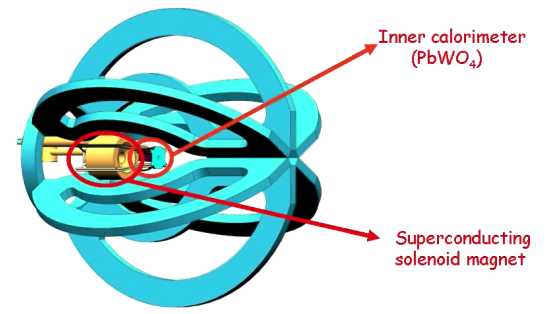

(a)

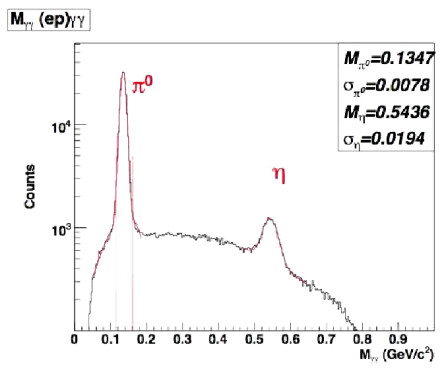

(b)

FIGURE 1. (a) The CLAS confi guration with new detector systems during the El-DVCS experiment with the newly installed inner calorimeter (IC) and superconducting solenoid. (b) Reconstructed invariant mass distribution from two photon clusters in IC.

The first part of E01-113, called "E1-DVCS", was conducted in 2005 with CLAS at Jefferson Lab. In order to ensure an accurate selection of exclusive channels, all finalstate particles produced were detected. The E1-DVCS collaboration built, commissioned and installed two new devices in CLAS, a superconducting solenoid magnet and a 424channel $\mathrm{PbWO}_{4}$ crystal inner calorimeter. The new inner calorimeter (IC) was installed to detect the photons at small angles from $5^{\circ}$ to $15^{\circ}$ in the laboratory frame. The new superconducting solenoid was designed to shield the CLAS drift chambers (DC) and the new IC from Møller electrons. The CLAS configuration with two new detector systems is shown in Figure 1(a). DVMP of $\pi^{0}$ and $\eta$ events were measured simultanously with DVCS events. The invariant mass distributions of two photon clusters in IC are shown in Figure 1(b).

The calibrations of E1-DVCS data are complete and the advanced stages of analysis are underway. After calibration of all detector subsystems and particle identification 


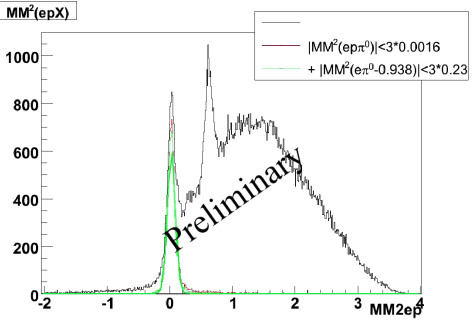

(a)

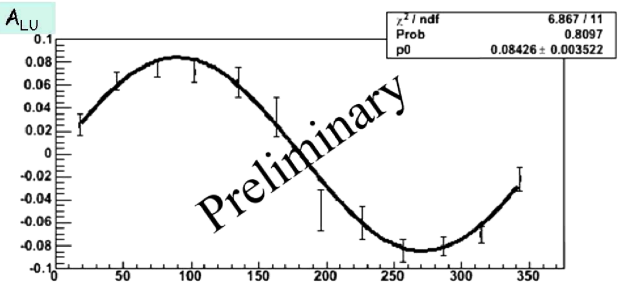

(b)

FIGURE 2. (a) Missing mass squared for $e p \rightarrow e p \gamma X$ for events with a good electron trigger, one proton, and one $\pi^{0}$ identifi ed by IC. The lowest (green) curve is obtained after various kinematic constraints are applied. (b) $A_{L U}$ in $\phi$ for the reaction $e p \rightarrow e p \pi^{0}$, integrated over all values of $Q^{2}, \mathbf{x}_{B}$ and $t$.

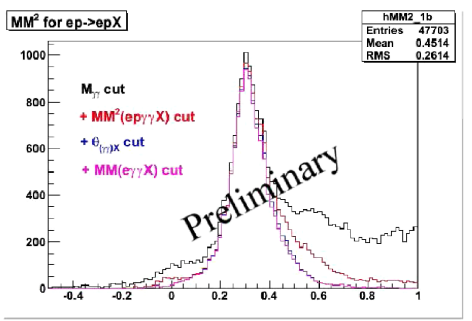

(a)

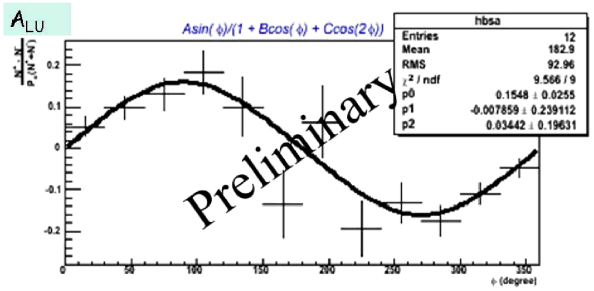

(b)

FIGURE 3. (a) Missing mass squared for $e p \rightarrow e p \gamma X$ for events with a good electron trigger, one proton, and one $\eta$ identifi ed by IC. The lowest (pink) curve is obtained after various kinematic constraints are applied. (b) $A_{L U}$ in $\phi$ for the reaction $e p \rightarrow e p \eta$, integrated over all values of $Q^{2}, \mathrm{x}_{B}$ and $t$

cuts for the scattered electron, proton and photon, the selection of the $e p \rightarrow e p \pi^{0}$ and $e p \rightarrow e p \eta$ events is straightforward. Figures 2(a) and Figures 3(a) show the missing mass squared $\left(M_{X}^{2}\right)$ spectra for the $e p \rightarrow e p X$ events after requiring an electron trigger, a proton and one $\pi^{0}$ or $\eta$ by a cut on the IC invariant mass spectrum as shown in Figure 1(b). The $e p \rightarrow e p \pi^{0}$ and $e p \rightarrow e p \eta$ events can be clearly selected once kinematical constraints are applied. After a cut on the missing mass squared spectrum, the beam spin asymmetries $\left(A_{L U}\right)$ can be extracted as shown in Figures 2(b) and Figures 3(b) for $\pi^{0}$ and $\eta$ channels, respectively. These non-zero asymmetries from both channels indicate the presence of an interference between longitudinal and transverse amplitudes in the production of these pseudoscalar mesons. The separation of the longitudinal and transverse parts of the cross section will require additional work.

The second part of E1-DVCS, and the other experiment E05-114, called "EG1DVCS", are planned to run in 2008. EG1-DVCS uses a CLAS longitudinally polarized hydrogen target. The target material to be used is a solid ammonia which is polarized via the dynamic nuclear polarization technique. This requires the target to be cooled down to $1 \mathrm{~K}$, as well as to be in a uniform $\left(\Delta B / B=10^{-4}\right)$ magnetic field of $5 \mathrm{~T}$. The expected 
polarization is $70-90 \%$, which has already been achieved in previous CLAS experiments with a polarized target. The magnetic field is supplied by two Helmholtz coils placed axially around the target. The Helmholtz coils also provide magnetic shielding from background due to the Møller electrons, which is an order of magnitude larger than the inelastic hadronic production rate. The magnetic shielding allows us to use the IC as used in E1-DVCS to measure photons at small angles.

These two expeirments will test whether we are in a regime where a direct interpretation of the results in terms of GPDs is possible, and will pave the way for the future experiments at the $12 \mathrm{GeV}$ upgraded CEBAF at Jefferson Lab.

\section{REFERENCES}

1. X. Ji, Phys. Rev. D55, 7114 (1997).

2. X. Ji, Phys. Rev. Lett. 78, 610 (1997).

3. A.V. Radyushkin, Phys. Lett. B380, 417 (1996).

4. A.V. Radyushkin, Phys. Rev. D56, 5524 (1997).

5. J.C. Collins, L. Frankfurt and M. Strikman, Phys. Rev. D56, 2982 (1997).

6. M. Diehl, Phys.Rep. 38841 (2003).

7. B. Burkert, L. Elouadhhiri, M. Garçon, and S. Stepanyan, Jefferson Lab Experiment E01-113.

8. A. Biselli, L. Elouadhhiri, K. Joo and S. Niccolai, Jefferson Lab Experiment E05-114. 